\title{
Analysis of Muscle Activity Utilizing Bench Presses in the AnyBody Simulation Modelling System
}

\author{
Zhongqiu Ji, Huihui Wang, Guiping Jiang, and Lin Li \\ College of P.E. and Sports, Beijing Normal University, Beijing 100875, China \\ Correspondence should be addressed to Guiping Jiang; jiang_guiping0401@126.com
}

Received 22 January 2016; Revised 28 March 2016; Accepted 19 April 2016

Academic Editor: Azah Mohamed

Copyright (c) 2016 Zhongqiu Ji et al. This is an open access article distributed under the Creative Commons Attribution License, which permits unrestricted use, distribution, and reproduction in any medium, provided the original work is properly cited.

\begin{abstract}
Using the AnyBody human modeling system with identical weights and varying grip distance $(40.0 \mathrm{~cm}, 50.0 \mathrm{~cm}$, and $60.0 \mathrm{~cm})$, the stress distribution for the pectoralis and the muscle of upper extremity during a bench press was simulated, and the surface myoelectricity (EMG) method was validated. Methods. The physical parameters driving the model of the human body were selected as weights of $35.0 \%$ (25.0 kg) and grip distances. Conclusion. The validation of AnyBody software was proved as a high validity by using EMG test of four muscles' activity compared to AnyBody software. During a bench press, the pectoralis major is the main muscle, the pectoralis major discharge increases with the height of barbell increases, and the pectoralis major discharge decreases as the short grip width increases. When the grip width equals the shoulder width, the value of pectoralis minor is lowest; when the grip width is smaller or larger than the shoulder width, the value is larger. As the short grip distance increases, the discharge of posterior deltoid muscle and triceps surface myoelectricity increases; thus, as the short grip distance increases, the deltoid muscle and triceps assist the pectoralis major during a bench press.
\end{abstract}

\section{Introduction}

Muscle strength is the ability of the body to complete a motion using muscle contractions to overcome a given resistance [1]. Because it is a critical aspect in human movement, muscle strength plays an important role in improving human health and athletic performance. Muscles are composed of bundles of slender, cylindrical arrangements of muscle cells, which are also called muscle fibers and are the basic unit of muscle function. Muscle mainly consists of the anterolateral group and the medial group, which allows the body to perform squats and sit-ups and also allows for bending and spinning of the body, amongst other actions [2, 3]. During training, muscle fibers can become enlarged, resulting in an increase in both the muscle meat's cross-sectional area and the muscle's capillary networks, thickened muscles and connective tissue, increased muscle glycogen, and so forth. These changes result in stronger muscle contractions. Scientifically based, efficient strength training is necessary to increase muscle strength, and scientific training methods attract significant amounts of attention from academic and professional sports teams worldwide $[4,5]$.
Most of the literature on muscle strength training illustrates methods to improve muscle strength using the physiological structure of muscles, improved training methods, and various training preparation activities as well as emphasizing relaxing after training and following a nutritious diet. Few studies have examined the muscle contraction characteristics of a specific strength training exercise $[6,7]$. In addition, only a few studies have examined the electrical characteristics of a specific muscle strength training activity using simulations of the human body, and the study of the scientific method of narration is also very general [8-13]. Therefore, a description and contrastive analysis concerning the aspects of human body surface myoelectricity characteristics and surface myoelectricity are conducted in this paper using an AnyBody simulation of a bench press strength training exercise. Based on basic principles of kinematic data and motor function and morphology, this paper analyzes the characteristics and functions of muscle structure and the discipline of collaboration characteristics using the specific action structure analysis and surface myoelectricity test methods. When the AnyBody simulation model is used to 
analyze strength training using bench presses, the activation and collaboration characteristics for varying grip distances using identical weight load percentages offer the data necessary for scientifically based strength training. This study tests the bench press exercise using a weight load of 35\% and grip widths corresponding to $40 \%, 50 \%$, and $60 \%$ of the subject's doubled shoulder widths.

Bench press is short for elevated bench press, also called a bench or lie-put-forward, and mainly trains the pectoralis major (a small chest muscle), deltoid toe, triceps, and elbow muscles, and is practiced before exercising the saw muscle, biceps, beak brachial muscle, forearm muscles, and so forth. Bench presses involve a number of muscles and have especially significant effects on the development of the upper limbs extensor and pectoralis major, an exercise that is unmatched by other exercises and therefore is known as the "king of exercises" [14]. Currently, there are a number of different types of strength training for different sports, where strength training utilizing bench presses has been adopted by a number of sports coaches [15-18]. Bench presses are one of the most basic actions in strength training and are a required exercise for athletes in professional power lifting contest. Bench presses are necessary for men's basketball players in Europe to improve their muscle strength control during rebounding. Similarly, bench presses are an essential physical exercise for firearm marksmanship practice $[19,20]$. The grip distance should be only slightly wider than the shoulders, which is required to be no larger than $81 \mathrm{~cm}$ in power lifting. During a bench press, an athlete's trunk cannot be twisted, and an equal amount of strength is required from both the left and right sides of the body. Moreover, the athlete's arms must be perpendicular to the trunk and parallel to the ground, their feet must be separated, and their hips and back cannot leave the bench.

\section{Methods}

Six male subjects, all 19 and 20 years of age, volunteered to participate in this study. Their average heights, weights, and shoulder widths were $175.0 \pm 3.0 \mathrm{~cm}, 71.4 \pm 2.2 \mathrm{~kg}$, and $50.0 \pm 3.0 \mathrm{~cm}$, respectively. The subjects were healthy freshman males that had passed the college entrance examination. The subjects did not have a history of severe trauma to their chest or nerve dysfunction. Before the experiment, the lengths of subject's trunks, upper arms, forearms, thighs, calves, and feet were measured to determine their main morphological indices.

Six subjects lifting $20 \mathrm{~kg}, 25 \mathrm{~kg}$, and $30 \mathrm{~kg}$ weight with varying short grips as a percentage of their twice shoulder breadth $(40 \%, 50 \%$, and $60 \%$ doubled shoulder width) completed 10 consecutive bench presses, which is the time required to record the process.

2.1. Model Construction. For this study, the standing model in the AnyBody 5.2 software package was modified according to the subjects' height, weight, and the average of their main morphological indices while considering their proportionality and fat percentages for the ScalingLengthMassFat function, and gravity was taken to be $9.81 \mathrm{~N} / \mathrm{kg}[21,22]$.
The contraction of muscles during motion cannot be accurately simulated because it is regulated by the central nervous system (CNS). Therefore, when the model needs to determine the actual muscle freedom required, redundancy issues may arise if a quantity that is greater than the number of muscles in the model is needed. AnyBody is used to provide the optimal solutions

$$
\begin{array}{ll}
\operatorname{Min} & \left(G\left(f^{M}\right)\right) \\
& G\left(f^{M}\right)=\sum_{i=1}^{n^{M}}\left(\frac{f_{i}^{M}}{N_{i}}\right)^{p} \\
& C f^{M}=d, \\
& 0 \leq f_{i}^{M} \leq N_{i}, \\
& i \in\left\{1, \ldots, n^{M}\right\} \leq N_{i}, \\
i \in\left\{1,2, \ldots, n^{M}\right\} .
\end{array}
$$

For the strength model, $G$ represents the objective function, which is the criterion of the recruitment of the CNS and minimized to all unknown forces in the activity. $C$ represents the system's coefficient matrix for the unknown forces, $d$ represents the applied loads and inertial force, $f^{M}$ is the muscle forces, and $N_{i}$ is muscle strength. Equation (2) is the polynomial criteria. Equation (3), as constraints to the optimization, is the dynamic equilibrium equation. Based on muscle fatigue, its activity is directly related to the assumption that the strength distribution follows the optimization criterion of minimizing the sum of the power $p$ of the muscle activity, guaranteeing a minimum level of fatigue, where $p=3$ [23].

The model was modified from the standing position to supine position with upper arm flexion and extension, and the average morphological indices of subjects were imported into the model (Figure 1). In this paper, six participants lifted weights corresponding to $30 \%$ (20 kg), 35\% (25 kg), and $35 \%$ to $40 \%(30 \mathrm{~kg})$ of their weight. The subjects had varying shoulder breadths and used varying grip widths (corresponding to $40 \%, 50 \%$, and $60 \%$ doubled shoulder width) during 10 consecutive bench presses. The time required to record the process and the time to complete the bench press were chosen as suitable for the relative loads used in sports and were used as physical parameters for the simulation. The pectoralis major muscle group is used to approximate the maximum force of the pectoralis major. The output of the pectoralis major force was found to vary during the exercise.

After multiple subject morphological input parameters, short grip distances, and loads, respective AnyBody model results and inverse dynamics analyses are obtained, and the five largest pectoralis major muscle force values are found. The model library in the AnyBody simulation software defines the pectoralis major muscle bundles in article 14: five muscle bundles (pectoralis major thoracic: 1-5) are connected to the front surface of manubrium mesosternum and the costal cartilage which start from the first to the sixth; five muscle bundles (pectoralis major clavicular: 1-5) are 

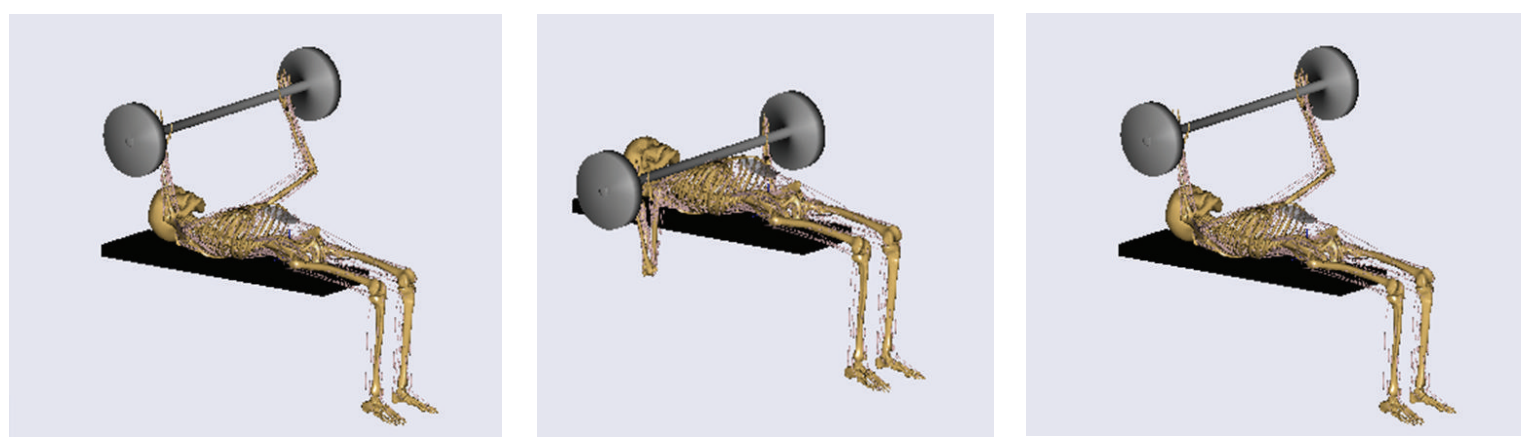

FIGURE 1: The three stages of the bench press.

connected to the inside of half collar bone; and the other four (pectoralis minor: 1-4) are connected to the costal cartilage which start from the third to the fifth and end with processus coracoideus scapulae. The power corresponding to the largest pectoralis major muscle bundle stress value is selected to represent the force of the pectoralis major.

2.2. The Surface Myoelectricity. The Noraxon Surface Electromyography test system records the EMG data of the subjects' upper arm movements, bow deltoids, right side of the right upper arm bicep's brachii, right upper limb triceps brachii, right side of the upper limb flexor carpi ulnaris, and trunk pectoralis major muscle. The sampling period is $3 \mathrm{~s}$, and the sampling frequency is $1000 \mathrm{~Hz}$. The original electromyographic signal is filtered (using a bandpass filter of $10-450 \mathrm{~Hz}$ ) and smoothed (RMS, $50 \mathrm{~ms}$ ), and the output of the five muscles with a $3 \mathrm{~s}$ integration is given as the electromyography (IEMG) value.

2.3. Data Statistics. Using the Noraxon software to analyze the surface myoelectricity data, the results were analyzed using SPSS17.0 with a single factor variance analysis and were given as $x \pm s$.

\section{Results}

3.1. Choosing 35\% Weights of the Human Body (25 kg) as a Relatively Suitable Load. Using $20 \mathrm{~kg}$ loads, six subjects completed 10 consecutive, quick bench presses at increasing speeds; using $30 \mathrm{~kg}$ loads, the six subjects were unable to complete 10 consecutive bench presses, indicating that the load is too large; using $35 \%$ ( $25 \mathrm{~kg}$ ) loads, the six subjects completed 10 consecutive bench presses using three short grip widths (corresponding to shoulder widths of $40 \%, 50 \%$, and $60 \%$ of their double shoulder breadths). This gives elbow angular speeds of $0.40 \pm 0.02^{\circ} / \mathrm{ms}, 0.43 \pm$ $0.07^{\circ} / \mathrm{ms}$, and $0.45 \pm 0.04^{\circ} / \mathrm{ms}$, respectively, and when completed at a moderate speed, a smaller average standard deviation is achieved indicating that the action is relatively stable. Based on the above discussion, a choice of $25 \mathrm{~kg}$ (35\% of weight) was made as an appropriate bench press load (Table 1).
TABLE 1: Average angular velocity for a complete arm flexion movement under different loads (unit: ${ }^{\circ} / \mathrm{ms}$ ).

\begin{tabular}{lccc}
\hline Weight $(\mathrm{kg})$ & \multicolumn{3}{c}{ Width distance $(\mathrm{cm})$} \\
\hline 20 & 40 & 50 & 60 \\
25 & $0.46 \pm 0.12$ & $0.51 \pm 0.08$ & $0.57 \pm 0.11$ \\
30 & $0.40 \pm 0.02$ & $0.43 \pm 0.07$ & $0.45 \pm 0.04$ \\
\hline
\end{tabular}

\subsection{The Simulation Results for the Human Body under a Load (25 kg)}

3.2.1. The Position Analysis of the Human Body Model. The subjects' forearm flex was separately completed 10 times. The time required to complete a bench press was recorded and indicated that the light $(20 \mathrm{~kg})$ load allowed the six subjects to complete their bench presses quicker. A load of $30 \mathrm{~kg}$ is too heavy of a load, and two subjects could not complete their bench presses. A load of $25 \mathrm{~kg}$ could be bench pressed by all six subjects. The bench press speed is relatively stable. A suitable load for athletes was chosen to be $25 \mathrm{~kg}$ (Figure 1).

An appropriate load $(25 \mathrm{~kg})$ using three short grip distances $(40 \mathrm{~cm}, 50 \mathrm{~cm}$, and $60 \mathrm{~cm}$ ) was input into the AnyBody simulation software as shown in Figure 1 and as shown in the simulation model. An inverse dynamics analysis is used to analyze the pectoralis major muscle bundle stress. For each parameter input to the AnyBody simulation, a load of $35 \%(25 \mathrm{~kg})$ and doubled shoulder widths corresponding to $40 \%(40 \mathrm{~cm}), 50 \%(50 \mathrm{~cm})$, and $60 \%(60 \mathrm{~cm})$ of the shoulder breadth are used as the experimental variables to drive the simulation of the human body.

3.2.2. The Major Pectoralis Muscle Force. Table 2 shows that the output force of pectoralis major thoracic in the three grip width is the largest between the three parts of pectoralis, pectoralis major clavicles take a second part, and both of the two-part muscles decrease with the grip width increase. The values of pectoralis minor at different grip width, respectively, are $114.0 \mathrm{~N}, 108.9 \mathrm{~N}$, and $136.6 \mathrm{~N}$. That is, when the grip width equals the width of shoulder, the value is lowest; when the grip 
TABLE 2: The major pectoralis muscle using varying grip widths with identical forces (unit: $N$ ).

\begin{tabular}{lccc}
\hline \multirow{2}{*}{ Name of the muscles } & \multicolumn{3}{c}{ Grip distance $(\mathrm{cm})$} \\
& 40 & 50 & 60 \\
\hline Pectoralis major thoracic 1 & 63.8 & 59.4 & 58.4 \\
Pectoralis major thoracic 2 & 59.4 & 54.8 & 52.8 \\
Pectoralis major thoracic 3 & 52.8 & 48.6 & 45.9 \\
Pectoralis major thoracic 4 & 47.0 & 43.2 & 40.9 \\
Pectoralis major thoracic 5 & 38.7 & 35.3 & 33.1 \\
Pectoralis major thoracic: 1-5 & 261.7 & 241.3 & 231.1 \\
Pectoralis major clavicular 1 & 34.3 & 31.4 & 29.4 \\
Pectoralis major clavicular 2 & 34.3 & 31.7 & 30.1 \\
Pectoralis major clavicular 3 & 34.5 & 32.5 & 31.1 \\
Pectoralis major clavicular 4 & 33.8 & 32.0 & 30.6 \\
Pectoralis major clavicular 5 & 34.5 & 33.4 & 33.3 \\
Pectoralis major clavicular: 1-5 & 171.4 & 161.0 & 154.5 \\
Pectoralis minor 1 & 24.6 & 23.4 & 28.3 \\
Pectoralis minor 2 & 28.8 & 27.4 & 33.9 \\
Pectoralis minor 3 & 30.6 & 29.1 & 36.8 \\
Pectoralis minor 4 & 30.0 & 29.0 & 37.6 \\
Pectoralis minor: 1-4 & 114.0 & 108.9 & 136.6 \\
General force & 547.1 & 511.2 & 522.2 \\
\hline
\end{tabular}

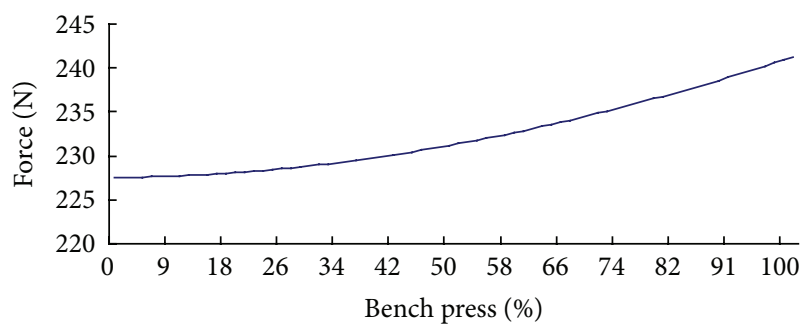

FIGURE 2: The change of the major pectoralis during bench presses.

width is smaller or larger than the width of shoulder, the value of force is larger. And the largest force of pectoralis minor appears when the perpendicular distance between trunk and barbell is minimum.

3.2.3. The Change of the Major Pectoralis with $35 \%$ (25 kg) Weight of Body. The bench press exercise, which mainly involves bones and muscles, is obviously driven by the upper limb muscles and hindquarters cadres pectoralis major [24]. In the AnyBody simulation analysis, the five major pectoralis major thoracic muscles show similar force trends, where the force of the pectoralis major reflects the stress of the pectoralis major changes. As shown in Figure 2, one time of bench press was normalized. A normalized bench press is from the barbell at the lowest station to the highest position. Obviously pectoralis major strength increases with the rising of the barbell.

3.2.4. Analysis of the Major Pectoralis's Force Using Three Grips. Under a load corresponding to a $35 \%$ weight $(25 \mathrm{~kg})$, using
TABLE 3: The major muscles using varying grip widths with identical forces of $25 \mathrm{~kg}$ (unit: $N$ ).

\begin{tabular}{lccc}
\hline \multirow{2}{*}{ Name of the muscles } & \multicolumn{3}{c}{ Grip distance $(\mathrm{cm})$} \\
& 40 & 50 & 60 \\
\hline Right biceps brachii & 9.39 & 6.33 & 9.38 \\
Right triceps brachii & 86.11 & 74.93 & 71.65 \\
Right posterior deltoid & 40.49 & 44.81 & 55.17 \\
Right pectoralis major thoracic & 261.7 & 241.3 & 231.1 \\
\hline
\end{tabular}

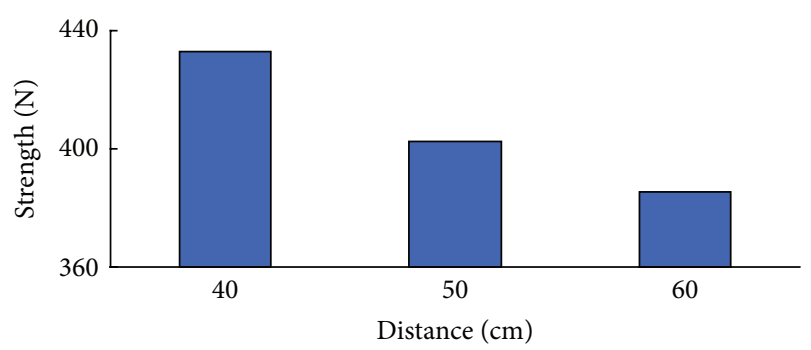

FIGURE 3: Analysis of the major pectoralis force using three grips.

doubled shoulder widths corresponding to $40 \%(40 \mathrm{~cm})$, $50 \%(50 \mathrm{~cm})$, and $60 \%(60 \mathrm{~cm})$ of the doubled shoulder breadth, three different grip distances were used to complete a bench press. According to Table 2, the pectoralis major plays an important role in bench press, the maximum value of pectoralis minor appears when the barbell lies in the lowest station. The pectoralis major force is found to be $433.1 \mathrm{~N}$ when the short grip distance is $40 \mathrm{~cm}$; when the short grip distance is decreased to $50 \mathrm{~cm}$, the stress is $402.3 \mathrm{~N}$. When the short grip is increased to $60 \mathrm{~cm}$ the grip force is $385.6 \mathrm{~N}$ for the pectoralis major muscle groups; the force decreases with the grip distance increases. When the short grip distance is small, strong contractions of the pectoralis major occur resulting in higher activity and a larger strength output (Figure 3 ).

3.2.5. The Major Muscle Force during Arm Extension Simulated by Modelling System. Based on a biomechanical simulation, Table 3 shows that the pectoralis major discharge increases as the short grip distance gradually decreases but still accounts for a majority of the muscle's strength. The triceps decreases with the grip width increase, and the posterior deltoid is on the opposite, which indicates that the posterior deltoid muscle and triceps are a major support work to the pectoralis major muscle during a bench presses' process.

\subsection{Verification Using Surface Electromyography Methods}

3.3.1. Muscle Electromyography MVC Ratio (\%) for Five Complete Bench Presses. Each subject completed 10 consecutive bench presses using arms to initially support the barbell at nearest distance of chest and subsequently raise the barbell until their arms were fully extended. Before the test, $25 \mathrm{~kg}$ dumbbell tests for the five largest surface myoelectric signals during isometric muscle contraction were analyzed and 
TABLE 4: Muscle electromyography mean/maximum average ratio (\%) for four complete bench presses.

\begin{tabular}{lccc}
\hline \multirow{2}{*}{ Name of the muscles } & 40 & 50 & 60 \\
\hline Right biceps brachii & $9.09 \pm 2.13$ & $6.61 \pm 1.61$ & $9.90 \pm 1.58$ \\
Right triceps brachii & $14.67 \pm 0.53$ & $12.74 \pm 3.90$ & $9.27 \pm 1.63$ \\
$\begin{array}{l}\text { Right posterior deltoid } \\
\begin{array}{l}\text { Right pectoralis major } \\
\text { thoracic }\end{array}\end{array}$ & $8.54 \pm 0.91$ & $10.62 \pm 4.19$ & $13.94 \pm 0.84$ \\
\hline
\end{tabular}

TABLE 5: Four muscles' MVC\% (\%).

\begin{tabular}{lccc}
\hline Name of the muscles & \multicolumn{3}{c}{ Grip distance $(\mathrm{cm})$} \\
& 40 & 50 & 60 \\
\hline $\begin{array}{l}\text { Right biceps brachii } \\
10.30 \pm 1.46\end{array}$ & $8.90 \pm 2.34$ & $11.40 \pm 1.81$ \\
$\begin{array}{l}\text { Right triceps brachii } \\
26.40 \pm 1.05\end{array}$ & $11.50 \pm 1.64^{* *}$ & $10.35 \pm 0.67^{* *}$ \\
$\begin{array}{l}\text { Right posterior } \\
\text { deltoid }\end{array}$ & $7.00 \pm 1.12$ & $10.90 \pm 3.10^{*}$ & $24.80 \pm 0.67^{* * \# \#}$ \\
$\begin{array}{l}\text { Right pectoralis } \\
\text { major thoracic }\end{array}$ & $39.60 \pm 1.41$ & $29.10 \pm 1.52^{* *}$ & $26.30 \pm 1.99^{* * \#}$ \\
\hline
\end{tabular}

${ }^{*} P<0.05$ indicates significant difference compared to $40 \mathrm{~cm}$ at 0.05 level.

${ }^{* *} P<0.01$ indicates significant difference compared to $40 \mathrm{~cm}$ at 0.01 level.

${ }^{\#} P<0.05$ indicates significant difference compared to $50 \mathrm{~cm}$ at 0.05 level.

${ }^{\# \#} P<0.01$ indicates significant difference compared to $50 \mathrm{~cm}$ at 0.01 level.

marked as the muscle of maximum voluntary contraction (MVC). The mean square root electromyography (RMS) was recorded during the experiments. The result can be described by a ratio, that is, the RMS divided by the MVC, which can be used to quantify the muscle's strength. Table 4 shows that four muscles are involved in a bench press, and the pectoralis major, deltoid muscle, and triceps' average discharge are important to power muscles during a bench press.

3.3.2. Comparison of the Four Muscles' MVC Ratio (\%) during a Bench Press. The MVC\%, a quantity found by dividing the electrical peak by the muscle's isotonic contraction's electricity peak, reflects the muscles involved in a bench press, and it is concluded that the ratio can quantify the reaction muscle's activation levels. Table 5 shows the six subjects under $25 \mathrm{~kg}$ loads using three short grip distances to complete 10 bench presses and that the MVC\%, pectoralis major, and deltoid are at higher activation levels. Both the activation of the pectoralis major level and the short grip have a significant correlation. As seen in Figure 4, the pectoralis major power increases as the short grip distance gradually decreases but still accounts for a majority of the muscle's power. The triceps and deltoid provide the force for the grip as the short grip distance increases thus indicating that the deltoid muscle and triceps are a major auxiliary power source to the pectoralis major muscle during a bench presses' finish.

3.3.3. The Maximum Stress and Pectoralis Major MVC\% Change Analysis. During a bench press in the AnyBody simulation, the maximum stress of the pectoralis major reflects the pectoralis major force output; the $\mathrm{MVC} \%$ is found

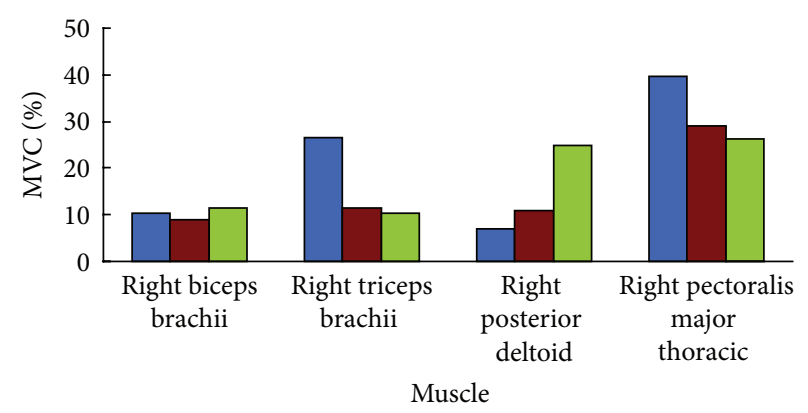

$\square 40$

口 50

ㅁ 60

FIGURE 4: Muscle size and short grip force differences.

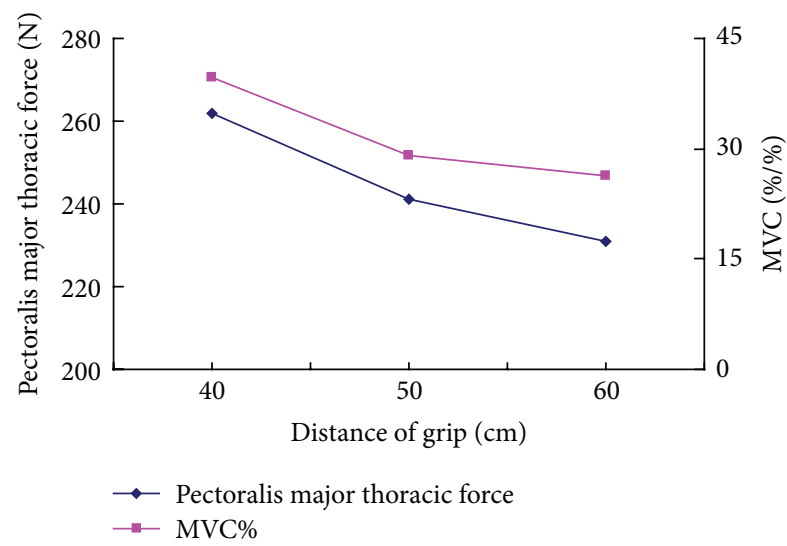

FIGURE 5: The relation between the maximum stress and pectoralis major MVC\% differences.

before the muscle contraction to determine the change in the electricity peak ratio, discharge level, and degree of muscle activation. As seen in Figure 5, the maximum stress and pectoralis major MVC\% are closely related (Correlation Index: $R=0.991, P<0.05)$ and can respond to changes in the pectoralis major during the bench press. Using $40 \mathrm{~cm}$, $50 \mathrm{~cm}$, and $60 \mathrm{~cm}$ short grips during a bench press, the maximum stress of pectoralis major thoracic and pectoralis major MVC\% values decreases with increasing short grip, which confirms the observation that the pectoralis major force output increases with decreasing short grip distance, and the pectoralis major activation level decreases with increasing short grip.

3.3.4. Integral Electromyographic Activity Values Using Varying Short Grips. RMS refers to the amplitude of period of the electromyography curve's envelope, to a certain extent, and characterizes the motor unit during a certain time period during a complete discharge [12].

During a bench press, the time integral of pectoralis major muscle electricity value is used to quantify the value of the muscle discharge, which reflects the level and discharge of the pectoralis major. As seen in Figure 6, the six subjects' IEMG values are larger, which explains why the pectoralis 


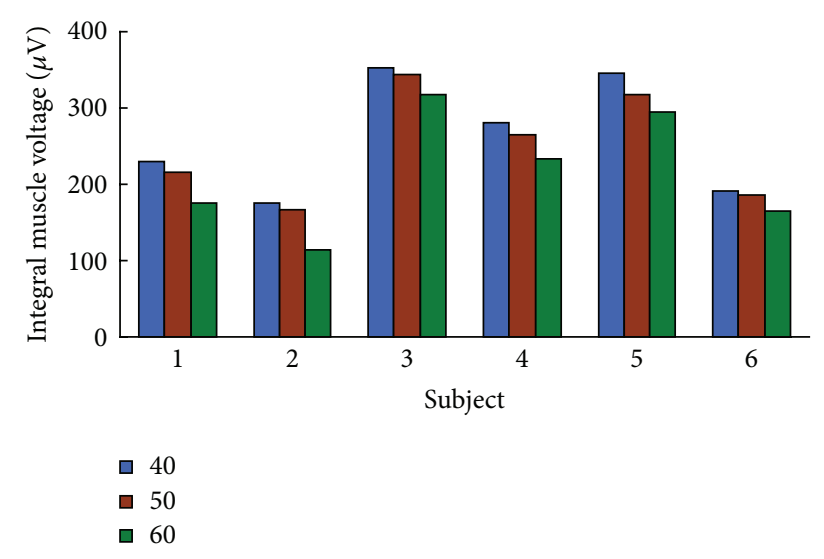

FIGURE 6: Subjects' IEMG values for the major pectoralis using three different grips.

major is one of the major muscles involved in a bench press. The six subjects' integral electrical values decrease with increasing short grip distance; the discharge of pectoralis major indicates a reduced force output.

\section{Conclusion}

Different loads affect a muscle's strength during bench presses, which affects the training efficiency and the athlete's resulting fitness levels. Thus, it is crucial to determine an appropriate training load for a bench press.

Varying the short grip distance results in a varying force output of the pectoralis muscle group during a bench press. As the short grip distance increases the pectoralis major produces less force during a bench press. The grip force is $433.1 \mathrm{~N}$ for the pectoralis major muscle groups at a width of $40 \mathrm{~cm}$; when the short grip is increased to $50 \mathrm{~cm}$, the stress decreases to $402.3 \mathrm{~N}$. The short grip force is $385.6 \mathrm{~N}$ when the short grip width is increased to $60 \mathrm{~cm}$. With the increase of barbell height, the discharge of pectoralis major increases. The values of pectoralis minor at different grip, respectively, are $114.0 \mathrm{~N}, 108.6 \mathrm{~N}$, and $136.6 \mathrm{~N}$. When the grip width equals the shoulder width, the value of pectoralis minor is lowest; when the grip width is smaller or larger than the shoulder width, the value is larger.

During a bench press, the posterior deltoid muscle and triceps are major support to the major muscle. This result between AnyBody software and Surface Electromyography is the same, which proved high validity for AnyBody software.

During a bench press, the pectoralis major discharge increases with the height of barbell increases, and the pectoralis major surface myoelectricity value gradually decreases as the short grip width increases. As the short grip distance increases, the discharge of posterior deltoid muscle and triceps surface myoelectricity increases; thus, as the short grip distance increases, the deltoid muscle and triceps assist the pectoralis major during a bench press.

\section{Competing Interests}

The authors declare that they have no competing interests.

\section{Acknowledgments}

This work was funded by the Ministry of Education of Humanities and Social Science Project (11YJA880037).

\section{References}

[1] R. D. Crowninshield and R. A. Brand, "A physiologically based criterion of muscle force prediction in locomotion," Journal of Biomechanics, vol. 14, no. 11, pp. 793-801, 1981.

[2] D. T. Davy and M. L. Audu, "A dynamic optimization technique for predicting muscle forces in the swing phase of gait," Journal of Biomechanics, vol. 20, no. 2, pp. 187-201, 1987.

[3] M. Damsgaard, J. Rasmussen, S. T. Christensen, E. Surma, and M. de Zee, "Analysis of musculoskeletal systems in the AnyBody Modeling System," Simulation Modelling Practice and Theory, vol. 14, no. 8, pp. 1100-1111, 2006.

[4] C. Robert, "Functional fatigue of the hip and ankle musculature cause similar alterations in single leg stance postural control," Journal of Science and Medicine in Sport, vol. 13, pp. 161-166, 2010.

[5] T. Field, "Tai Chi research review," Complementary Therapies in Clinical Practice, vol. 17, no. 3, pp. 141-146, 2011.

[6] S. L. Delp and J. P. Loan, "A computational framework for simulating and analyzing human and animal movement," IEEE Computing in Science and Engineering, vol. 2, no. 5, pp. 46-55, 2000 .

[7] S. L. Delp, J. P. Loan, M. G. Hoy, F. E. Zajac, E. L. Topp, and J. M. Rosen, "An interactive graphics-based model of the lower extremity to study orthopaedic surgical procedures," IEEE Transactions on Biomedical Engineering, vol. 37, no. 8, pp. 757767, 1990.

[8] F. B. Horak, "Clinical measurement of postural control in adults," Physical Therapy, vol. 67, no. 12, pp. 1881-1885, 1987.

[9] I. H. J. Logghe, A. P. Verhagen, A. C. H. J. Rademaker et al., "The effects of Tai Chi on fall prevention, fear of falling and balance in older people: a meta-analysis," Preventive Medicine, vol. 51, no. 3-4, pp. 222-227, 2010.

[10] V. Mohammadi, M. Alizadeh, and A. Gaieni, "The effects of six weeks strength exercises on static and dynamic balance of young male athletes," Procedia-Social and Behavioral Sciences, vol. 31, pp. 247-250, 2012.

[11] M. L. Madigan, B. S. Davidson, and M. A. Nussbaum, "Postural sway and joint kinematics during quiet standing are affected by lumbar extensor fatigue," Human Movement Science, vol. 25, no. 6, pp. 788-799, 2006.

[12] T. Paillard, E. Margnes, J. Maitre et al., "Electrical stimulation superimposed onto voluntary muscular contraction reduces deterioration of both postural control and quadriceps femoris muscle strength," Neuroscience, vol. 165, no. 4, pp. 1471-1475, 2010.

[13] W. R. Taylor, M. O. Heller, G. Bergmann, and G. N. Duda, "Tibio-femoral loading during human gait and stair climbing," Journal of Orthopaedic Research, vol. 22, no. 3, pp. 625-632, 2004.

[14] J. Dul, G. E. Johnson, R. Shiavi, and M. A. Townsend, "Muscular synergism-II. A minimum-fatigue criterion for load sharing between synergistic muscles," Journal of Biomechanics, vol. 17, no. 9, pp. 675-684, 1984.

[15] M. de Zee, L. Hansen, C. Wong, J. Rasmussen, and E. B. Simonsen, "A generic detailed rigid-body lumbar spine model," Journal of Biomechanics, vol. 40, no. 6, pp. 1219-1227, 2007. 
[16] M. G. Pandy, "Computer modeling and simulation of human movement," Annual Review of Biomedical Engineering, vol. 3, pp. 245-273, 2001.

[17] N. B. Singh, M. A. Nussbaum, and M. A. Madigan, "Evaluation of circumferential pressure as an intervention to mitigate postural instability induced by localized muscle fatigue at the ankle," International Journal of Industrial Ergonomics, vol. 39, no. 5, pp. 821-827, 2009.

[18] U. Granacher, M. Gruber, D. Förderer, D. Strass, and A. Gollhofer, "Effects of ankle fatigue on functional reflex activity during gait perturbations in young and elderly men," Gait \& Posture, vol. 32, no. 1, pp. 107-112, 2010.

[19] D. G. Thelen, F. C. Anderson, and S. L. Delp, "Generating dynamic simulations of movement using computed muscle control," Journal of Biomechanics, vol. 36, no. 3, pp. 321-328, 2003.

[20] C. Disselhorst-Klug, T. Schmitz-Rode, and G. Rau, "Surface electromyography and muscle force: limits in sEMG-force relationship and new approaches for applications," Clinical Biomechanics, vol. 24, no. 3, pp. 225-235, 2009.

[21] E. J. Bisson, D. McEwen, Y. Lajoie, and M. Bilodeau, "Effects of ankle and hip muscle fatigue on postural sway and attentional demands during unipedal stance," Gait \& Posture, vol. 33, no. 1, pp. 83-87, 2011.

[22] B. J. Fregly and F. E. Zajac, "A state-space analysis of mechanical energy generation, absorption, and transfer during pedaling," Journal of Biomechanics, vol. 29, no. 1, pp. 81-90, 1996.

[23] J.-B. Mao, X.-H. Jia, R.-C. Wang, F. Pu, and F. Sun, "Neck muscle activity during head flexion," Journal of Medical Biomechanics, vol. 27 , no. 5 , pp. 577-581, 2012.

[24] P. Worsley, M. Stokes, and M. Taylor, "Predicted knee kinematics and kinetics during functional activities using motion capture and musculoskeletal modelling in healthy older people," Gait \& Posture, vol. 33, no. 2, pp. 268-273, 2011. 


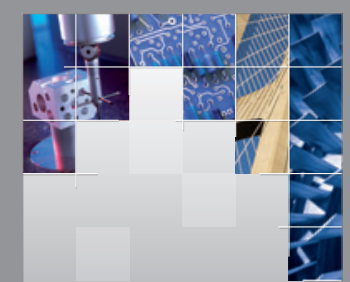

\section{Enfincering}
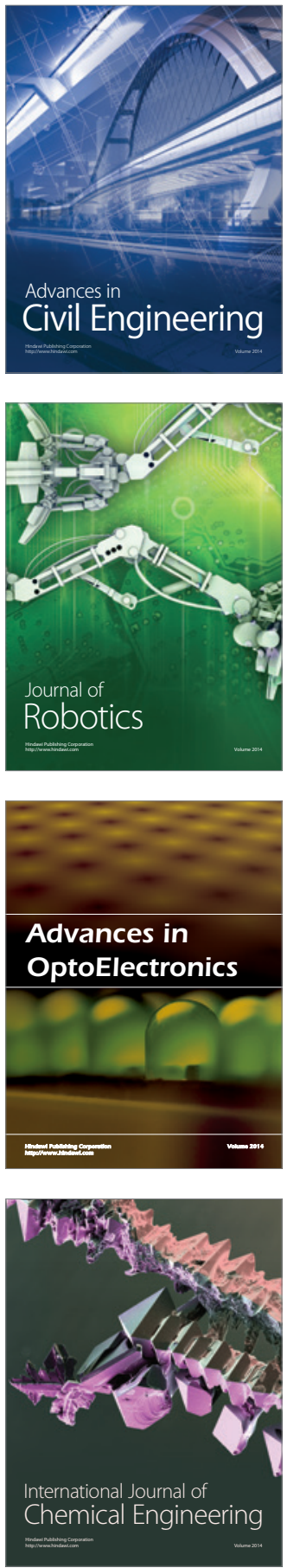

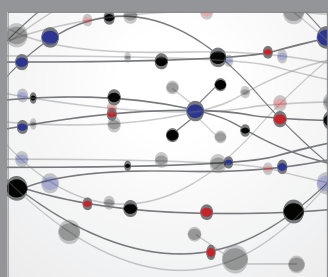

The Scientific World Journal

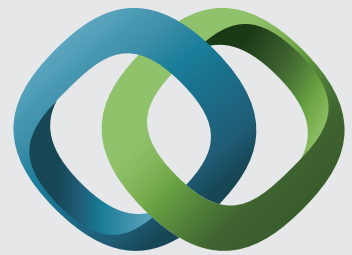

\section{Hindawi}

Submit your manuscripts at

http://www.hindawi.com
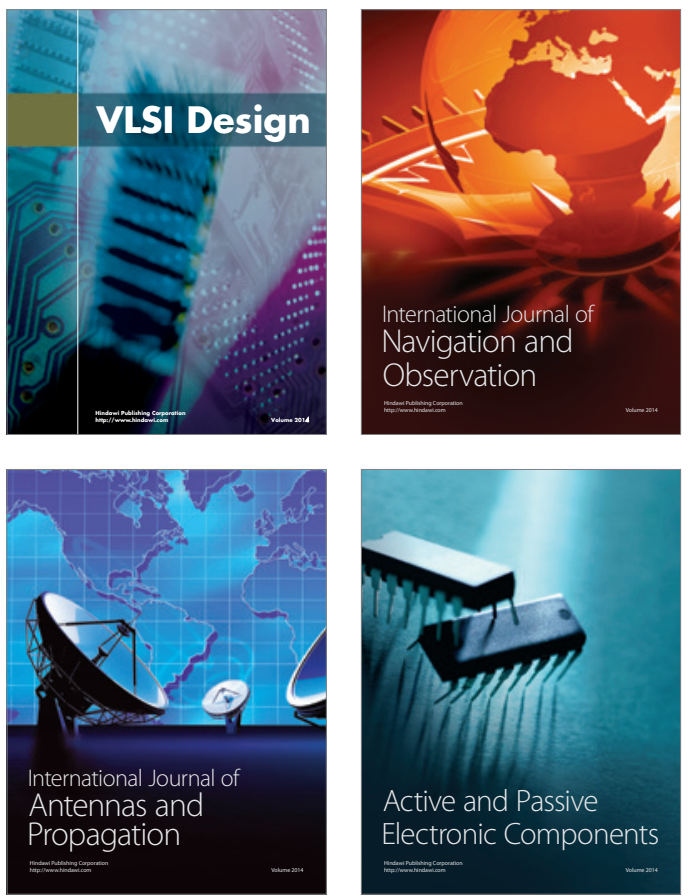
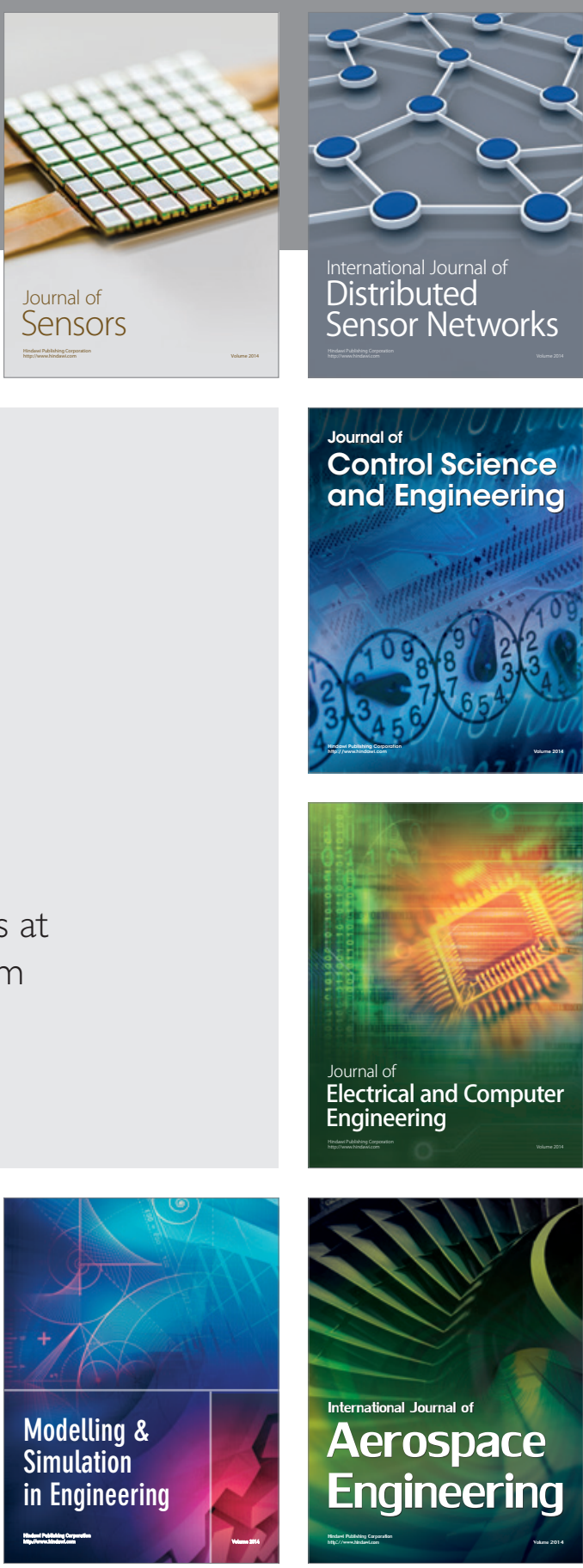

International Journal of

Distributed

Sensor Networks

Journal of

Control Science

and Engineering
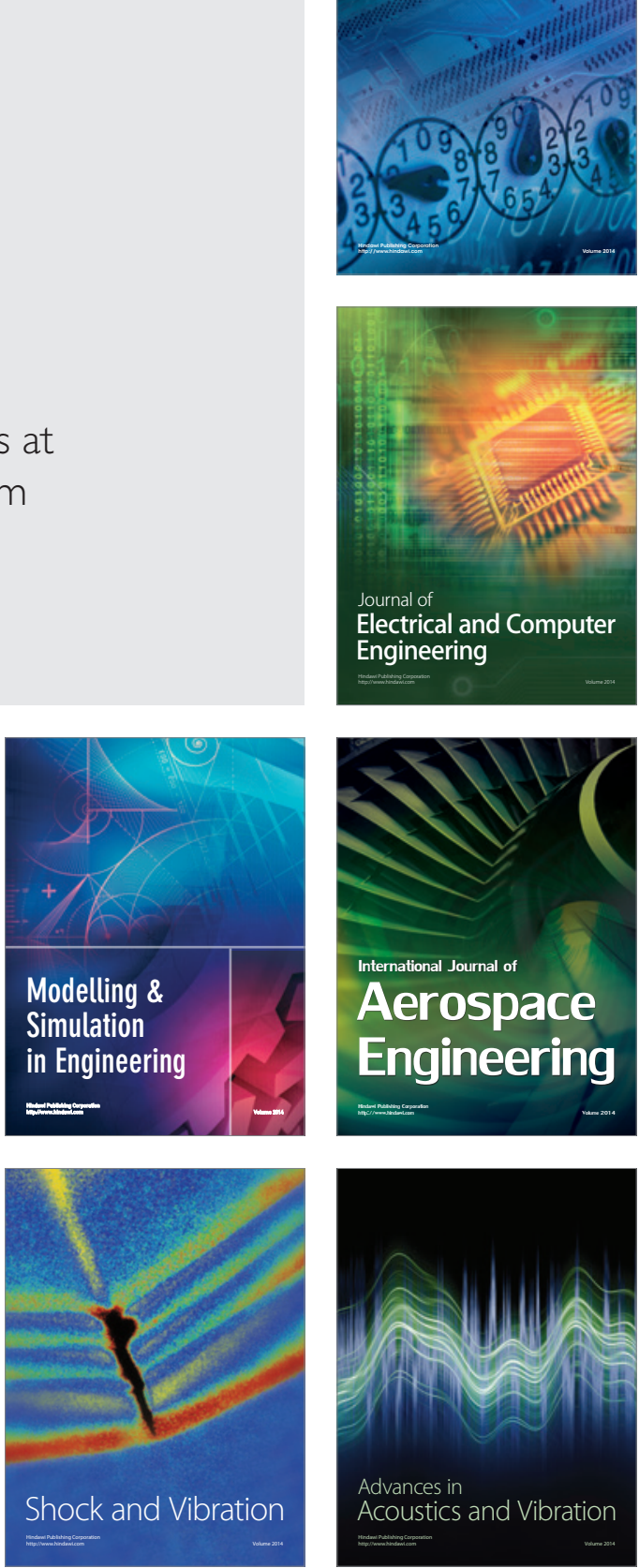